\title{
Oppositions kantiennes / polarités warburgiennes.
}

\section{Audrey Rieber}

\section{OpenEdition}

\section{Journals}

Édition électronique

URL : http://journals.openedition.org/imagesrevues/2845

DOI : 10.4000/imagesrevues. 2845

ISSN : 1778-3801

\section{Éditeur :}

Centre d'Histoire et Théorie des Arts, Groupe d'Anthropologie Historique de l'Occident Médiéval, Laboratoire d'Anthropologie Sociale, UMR 8210 Anthropologie et Histoire des Mondes Antiques

\section{Référence électronique}

Audrey Rieber, «Oppositions kantiennes / polarités warburgiennes. », Images Re-vues [En ligne], Horssérie 4 | 2013, document 4, mis en ligne le 30 janvier 2013, consulté le 31 janvier 2021. URL : http:// journals.openedition.org/imagesrevues/2845; DOI : https://doi.org/10.4000/imagesrevues.2845

Ce document a été généré automatiquement le 31 janvier 2021.

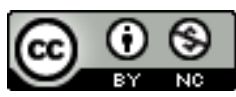

Images Re-vues est mise à disposition selon les termes de la Licence Creative Commons Attribution -

Pas d'Utilisation Commerciale 4.0 International. 


\title{
Oppositions kantiennes / polarités warburgiennes.
}

\author{
Audrey Rieber
}

1 Il est devenu courant d'éclairer la pensée de Warburg en la mettant en rapport avec la philosophie nietzschéenne, la psychanalyse, l'anthropologie ou encore la théorie des émotions de Darwin. Pour fructueuses que soient ces associations, c'est néanmoins sous un angle différent, néokantien, que j'envisagerai ici les «formules véritablement antiques

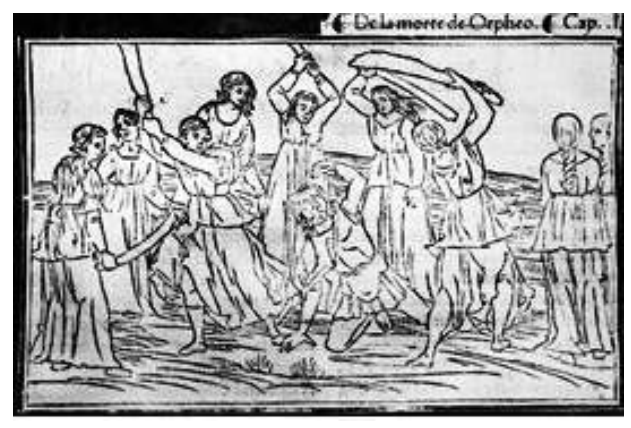
servant à l'expressivité exagérée du corps ou de l'âme $»^{1}$. Je ferai l'hypothèse que ces Pathosformeln et la structure psychique dont elles sont le symptôme peuvent être vues comme la réponse warburgienne au problème de la conciliation des oppositions d'entendement, qu'elles sont, en d'autres termes, une façon de « refuse[r] dans tous les domaines la pédanterie paralysante du « ou bien - ou bien », [non] pas faute de ressentir les contradictions dans toute leur acuité, mais par refus de les considérer comme irréductibles $»^{2}$. Non seulement les formules du pathos unissent des éléments en tension, mais la structure psychique dont elles sont le symptôme articule plusieurs pôles que les «abstractions de l'entendement séparateur", pour reprendre la formule hégélienne, pensent isolément ${ }^{3}$. En caractérisant le psychisme de schizophrène, Warburg met en circulation le sujet et l'objet, l'identité et la différence ou encore l'être et la pensée. Quant au couple Apollon et Dionysos, il permet lui aussi de penser les rapports entre les opposés sur un mode inédit qui scelle également la spécificité de Warburg par rapport à Nietzsche. L'hypothèse selon laquelle ces formules, en tant que symptômes d'un mouvement psychique oscillatoire entre des pôles que l'entendement est enclin à séparer, proposent une solution au problème postkantien de la conciliation des opposés peut évidemment surprendre, puisque c'est généralement l'iconologie panofskienne, et non la pratique psycho-historique de Warburg, que l'on inscrit dans un horizon 
postkantien, et plus précisément cassirérien. Mais le propos ne sera pas d'affirmer que Warburg a attribué pour fonction aux formules du pathos de penser ensemble ce que le kantisme sépare - les problèmes qu'il se pose ne sont pas ceux de Hegel, de Fichte ou de Cassirer. Il s'agira bien plutôt de montrer la portée spéculative des Pathosformeln que l'on peut inscrire dans le cadre $d u$ problème philosophique central introduit par la critique, la levée du dualisme kantien qui traverse aussi bien l'idéalisme allemand, la phénoménologie de Merleau-Ponty (l'être-au-monde) que la pensée de Deleuze et Guattari (la machine désirante). À quoi s'ajoute que les formules du pathos offrent non seulement une solution au problème de la mise en rapport des oppositions kantiennes, mais que, en pensant leur articulation dans le temps en termes non de téléologie ou de dialectique, mais d'oscillation, de battement ou de greffe, Warburg propose une conception inédite de la temporalité (historique).

\section{Figures de conciliation: Aufhebung, formes symboliques, Pathosformeln.}

2 Avant de montrer de quelle manière les formules du pathos font éclater ou, ce qui revient au même, font se réunir, les oppositions kantiennes, il n'est peut-être pas inutile de rappeler de quelle situation héritent les successeurs de Kant. Pour asseoir l'objectivité du jugement (scientifique) ainsi que pour libérer la philosophie des spéculations dogmatiques, Kant distingue fermement ce qui relève de l'objet de ce qui relève du sujet, ce qui est de l'ordre de l'être de ce qui est de l'ordre de la pensée, le concept de noumène jouant ici une place centrale. L'activité du sujet transcendantal consiste à structurer le donné mais sans le constituer. L'être, la matière demeurent ontologiquement et épistémologiquement extérieurs au sujet. Le sujet transcendantal impose une forme au fond (une distinction lourde de conséquences dans le domaine de l'art) sans qu'il y ait à proprement parler d'adéquation entre eux et sans qu'ils forment une véritable union. Lorsque l'entendement saisit la nature à travers les catégories, la connaissance ne se confond pas avec l'être. Les objets sont connaissable (on peut formuler à leur endroit un ensemble de jugements synthétiques a priori), mais leur structure ontologique demeure inaccessible. En plus de poser un écart irréductible entre le sujet et l'objet, l'être et la pensée, le criticisme kantien distingue les pouvoirs et les limites respectifs de nos facultés (raison, entendement, sensibilité, imagination). En soutenant que le sujet appréhende l'objet à l'aide de catégories a priori et d'une donation sensible structurée par les formes a priori de la sensibilité de l'espace et du temps, Kant garantit la possibilité de la science tout en marquant clairement le point à partir duquel l'entendement s'égare. Quant à la détermination des conditions d'un recours régulé aux idées, elle permet de marquer le point où l'usage de la raison devient dogmatique.

Mais cette double conquête se fait au prix de l'instauration d'une série d'oppositions que les successeurs de Kant s'efforceront de (ré)concilier. Il s'agit pour eux de dépasser la liberté formelle de l'entendement, c'est-à-dire, pour parler en termes hégéliens, d'aller au-delà de la « liberté de séparation d'avec l'être » qui consiste à « absolutiser ce qui la délimite, la détermine, la nie $»^{4}$. C'est dans ce contexte que j'aimerais voir dans les formules du pathos une réponse au problème postkantien de la conciliation des dualismes introduits par l'entendement séparateur. 
4 À l'instar de la forme symbolique que Cassirer, puis Panofsky, définissent comme une « énergie de l'esprit par laquelle un contenu de signification spirituelle est accolé à un signe sensible concret et intrinsèquement adapté à ce signe $»^{5}$, la formule du pathos, à sa manière, lève d'emblée et par principe l'opposition entre la forme et la sensibilité. Elle est à la fois formule, catégorie, mode de structuration et de production, fruit de l'activité d'un sujet créateur et sensibilité, affection, affectivité liée à une corporéité, passivité. Et, lorsque Warburg considère que "la rhétorique musculaire de Pollaiolo " est constituée "des superlatifs authentiquement antiques du langage des gestes ${ }^{6}$, il pense ensemble des catégories verbales, littéraires ("superlatifs", "rhétorique», «langage ») et des catégories corporelles ( geste », « musculaire ») : il met en rapport le verbe et la pensée avec le corps et la matière. C'est aussi l'opposition entre la forme et le contenu que les Pathosformeln rendent caduque, puisque les «modèles de mimiques pathétiques fortement accentuées $»^{7}$ sont non le signe mais le symptôme et le porteur de certains états psychiques. Tandis que le signe est dans un rapport extérieur et arbitraire à la chose qu'il désigne, le symptôme n'est pas un simple indicateur mais l'expression, la manifestation d'un état. Dans les formules du pathos, la forme n'est pas appliquée de l'extérieur au contenu; la forme n'est autre que le contenu qui s'est extériorisé. "Il n'y a aucune nécessité, lit-on dans la Phénoménologie de l'esprit, d'appliquer de l'extérieur au contenu concret le formalisme ; celui-là est, en lui-même, le passage dans celui-ci, lequel, cependant, cesse d'être un tel formalisme extérieur, parce que la forme est le devenir indigène du contenu concret lui-même $»^{8}$; le contenu est « quelque chose d'effectif, un sujet, ou un advenir à soi-même »'. Cette réflexion de Hegel, qui montre l'insuffisance d'une conception extérieure des rapports entre la forme et le fond, indique que les Pathosformeln comportent des enjeux philosophiques qui sont ceux de la philosophie postkantienne. Mais la solution de Warburg se distingue fondamentalement de celle de Hegel, puisque le « contenu » en question n'est pas l'idée mais un mélange inextricable d'états corporels, d'émotions et de pensées.

Tout aussi anti-kantienne est l'affirmation selon laquelle la forme est mouvante. « Là où d'autres avaient vu des formes déterminées délimitées, des formes reposant en ellesmêmes, il [Warburg] voyait des forces mouvantes, il voyait ce qu'il appelait les grandes «formes du pathos $»^{10}$.

6 Dans ce discours prononcé à l'occasion de la mort de son ami, Cassirer insiste sur la fluidité des catégories warburgiennesnes. Tandis que, pour Kant, l'imposition de formes par les catégories a pour fonction de structurer le devenir, d'arrêter le flux mouvant des phénomènes, bref, et selon les mots de la Critique de la raison pure, d'organiser la " rhapsodie des perceptions ", l' " exagération gestuelle » que Warburg repère chez un Botticelli n'est pas une simple mise en forme du mouvement mais une forme en mouvement, une mise en mouvement de la forme ${ }^{11}$. Ces gestes, loin d'être de simples formules esthétiques (au sens où l'on parle de "formules toutes faites ») sont donc dotés (et issus) d'une poussée générative. La force qui s'y manifeste est anachronique : les « superlatifs du langage mimique », porteurs d'une « capacité expressive idéalement intensifiée $»^{12}$, sont les symptômes de la disposition psychique fondamentale de l'homme. En la qualifiant de schizophrène, Warburg fournit un nouveau paradigme pour penser ensemble une série d'oppositions kantiennes entre le sujet et l'objet, l'être et la pensée, l'identité et la différence. 


\section{Catégories et schizophrénie.}

7 Le psychisme humain, animé, parfois secoué, d'une tension insoluble entre forces opposées, est qualifié par Warburg de schizophrénique. «Souvent il me vient à l'esprit que, en tant que psycho-historien, je cherche à établir la schizophrénie de la civilisation occidentale, à partir de ses images par un réflexe autobiographique : la nymphe extatique (maniaque) d'un côté, et le dieu fluvial mélancolique (dépressif) de l'autre $»^{13}$. Ce travail de psycho-historien n'est pas sans faire penser à l'entreprise psychologique du Nietzsche de La Naissance de la tragédie qui déclare faire sienne « une question psychologique aussi difficile que l'origine de la tragédie chez les Grecs »" Psychologue-historien, Warburg se donne pour tâche de déceler « la forme stylisée en sublime tragique, susceptible d'exprimer des valeurs-limites de l'expression mimique et physionomique $»^{15}$. Ces valeurs-limites manifestent une énergie et une structure schizophréniques propres au psychisme de Warburg et, de façon beaucoup plus intéressante, à l'ensemble de l'humanité. «L'humanité toute entière est éternelle et de tout temps schizophrène $»^{16}$. Dans le passage précédemment cité, la polarité se noue entre la nymphe extatique (maniaque) et le dieu fluvial mélancolique (dépressif), c'està-dire entre une jeune figure féminine (le terme de nymphe étant tiré du grec ancien

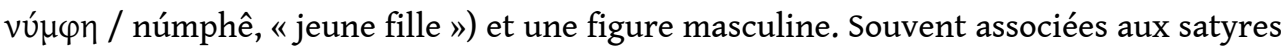
et à leur puissance sexuelle, les nymphes évoquent ici un débordement d'énergie qui s'oppose à la passivité mélancolique du dieu du fleuve. L'extase, le fait d'être hors de soi, extraverti, projeté à l'extérieur s'oppose bien à la dépression, au repli sur soi, à l'inhibition, bref à l' «abaissement" mélancolique, à son «enfoncement» pour reprendre l'étymologie latine de «depressio».

8 La manie, que Warburg associe à juste titre à l'extase, peut être définie comme un état de surexcitation où un intérêt exclusif est porté à certains objets. Elle est opposée à la mélancolie, caractérisée, elle, par un retrait de la libido, un abattement physique et moral. Les polarités mises en tension sont donc au nombre de trois; elles se nouent entre le féminin et le masculin, l'extérieur et l'intérieur, l'activité et la passivité. Il est important de comprendre qu'elles signifient moins deux possibilités antinomiques de l'esprit humain que les deux extrémités entre lesquelles il se meut. La schizophrénie, donc, permet de penser ensemble plusieurs séries d'oppositions entérinées par le kantisme, entre le sujet et l'objet, l'être et la pensée ou encore l'identité et la différence - une opposition dont Hegel a montré combien la suppression est nécessaire pour penser l'unité. La schizophrénie est en effet, par définition, une confusion entre le sujet

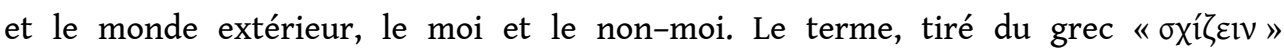
(fractionnement) et « $\varphi \rho \eta ́ v »$ (esprit), signifie littéralement « esprit coupé », clivage. C'est une "coupure de l'esprit", non au sens d'une personnalité double, comme on le croit le plus souvent, mais d'une perte de contact avec la réalité. D'un point de vue psychanalytique, elle résulte d'un conflit entre le Moi et la réalité ; en termes hégéliens, elle est l'identité de l'identité et de la différence. Warburg ne conçoit pas le rapport entre ces pôles d'une manière dialectique, comme pourrait le faire Hegel, mais sur le mode du battement, l'état schizophrène étant un état oscillatoire. Bleuler, qui a introduit le terme, a décrit comment les patients oscillent entre une sensibilité exagérée et une anesthésie totale des sentiments. En considérant que sa mission » est de «fonctionner comme un sismographe de l'âme sur la ligne de partage entre les cultures $»^{17}$, Warburg voit dans sa propre schizophrénie le symptôme mais surtout le 
capteur et le révélateur des secousses schizophréniques, transhistoriques et transculturelles, de l'humanité.

\section{Apollon et Dionysos}

Ces secousses, ces tensions et unions provisoires peuvent aussi être pensées à partir du couple nietzschéen d'Apollon et de Dionysos. Celui-ci permet de clarifier la notion de Pathosformeln, mais aussi de saisir plus précisément le rapport entre des pôles que le logicien considère comme contradictoires et le psychologue en conflit. En s'appuyant sur la paire conceptuelle nietzschéenne, on peut en effet envisager leurs relations d'une façon qui n'est pas antinomique (Kant), mais pas davantage dialectique (Hegel) : en termes de combat ou, au contraire, d'union mystérieuse. Mais rappelons au préalable en quels termes Nietzsche caractérise l'apollinien et le dionysiaque dans les premières pages de La Naissance de la tragédie. Tandis que le royaume d'Apollon est celui des arts plastiques (et d'une partie de la poésie), Dionysos règne sur les arts non plastiques de la musique et de la danse. Warburg prend donc une certaine liberté par rapport à Nietzsche lorsqu'il qualifie de dionysiaque certains traits psycho-formels d'œuvres appartenant aux arts plastiques. C'est notamment le cas dans son commentaire d'une gravure d'après les Métamorphoses d'Ovide, La Mort d'Orphée (maître anonyme, Venise, 1497), où il entend l'écho des "sombres mystères de la légende de Dionysos ${ }^{18}$. Avant de commenter plus en détail son analyse, j'aimerais présenter encore un peu l'apollinien et le dionysiaque nietzschéens. L'opposition de ces deux forces est celle qui distingue le rêve, la belle apparence, de l'ivresse ${ }^{19}$. Au fondement de cette dualité réside la reconnaissance, ou au contraire la suppression, du principe d'individuation. Si l'apollinien est le règne de la "mesure », de la « délimitation ", du «calme tout de sagesse» qui, sur le plan moral, s'expriment par les principes du «Connais-toi toi-même » et du «Rien de trop ", c'est parce qu'il consacre le principe d'individuation. Le dionysiaque résulte au contraire de sa rupture, puisque c'est de la réconciliation de l'homme avec l'homme et de l'homme avec la nature que procèdent ces caractères $\mathrm{du}$ dionysiaque que sont l'ivresse et l'extase. La "démesure » dionysiaque est l'expression de l'» harmonie universelle » ou, en d'autres termes, de l'» Un originaire ». Dans ses chants et ses danses, c'est la "puissance artiste de la nature toute entière " qui s'exprime. Les rapports entre l'apollinien et le dionysiaque sont complexes, puisqu'il y a conditionnement réciproque, conflit mais aussi union. Sur un plan métaphysique, Apollon ne peut exister sans Dionysos, car la conscience apollinienne n'est rien d'autre que le voile qui dissimule le fond dionysiaque de l'être. Mais, inversement, «l'être véritable, l'un originaire, en tant qu'éternelle souffrance et contradiction, a besoin en même temps, pour sa perpétuelle délivrance, de la vision extatique et de l'apparence délectable » (N.T., p. 39), l'artiste étant « en quelque sorte ce médium par l'entremise duquel le seul sujet qui existe véritablement fête sa délivrance dans l'apparence » (ibid., p.47). Si Apollon ne peut être sans Dionysos, celuici ne peut exister sans celui-là. Par ailleurs, dans la mesure où ils constituent des forces opposées, ils mènent un perpétuel combat l'un contre l'autre, combat néanmoins émaillé de réconciliations provisoires. Si, dans une perspective kantienne, une réconciliation de l'être et du rêve ou encore de l'ivresse et de la maîtrise est inconcevable, Nietzsche considère que c'est justement aux «noces mystérieuses » qui ont suivi le « long combat » de l'olympien et du dionysiaque que l'on doit la naissance de la tragédie attique et du dithyrambe dramatique. Or il est possible d'avancer que, 
parmi les fruits de cette union agonistique, figurent les formules du pathos. Tandis que leur dimension pathique renvoie au dionysiaque, leur caractère formulaire évoque la mesure et la forme apolliniennes qui délimitent et contiennent le «torrent dionysiaque ». Tandis que l'union de l'apollinien et du dionysiaque permet à Nietzsche de penser la naissance de la tragédie et du dithyrambe dramatique, Warburg s'en sert pour concevoir la nature et la possibilité des formules du pathos. Et à la thèse de Nietzsche selon laquelle cette union caractérise in fine l'ère hellénique en général - «le dionysiaque et l'apollinien, se renforçant, par une série sans cesse renouvelée d'enfantements réciproques et de conflits, ont dominé l'être hellénique »-, fait écho l'affirmation de Warburg selon laquelle "les études sur les religions de l'antiquité gréco-romaine nous enseignent toujours plus à considérer que l'Antiquité est en quelque sorte symbolisée par un hermès bifrons d'Apollon et Dionysos $»^{20}$. Et si la Renaissance est une résurgence de l'Antique, il n'est pas étonnant d'y voir sourdre à nouveau le conflit entre le "titanesque », ou le «barbare ", et la « liberté vis-à-vis des émotions les plus sauvages »- les termes sont de Nietzsche. Le philosophe toutefois, contrairement à ce que fera Warburg, n'interprète pas la Renaissance à partir du couple Apollon et Dionysos, mais l'appréhende sous un angle axiologique, celui de la restauration d'une haute culture : «Il y eut à la Renaissance un renouveau, brillant et extraordinaire, de l'idéal classique et du mode noble d'évaluation de toutes choses $»^{21}$. Warburg au contraire insiste sur le fait que le Quattrocento voyait dans l'Antiquité un "hermès au double visage, l'un sombre et démonique [ ...] et l'autre clair et olympien ${ }^{22}$. Quant à l'article consacré à "Albert Dürer et l'Antiquité italienne», il établit comment, " par l'intermédiaire des Italiens, il [Dürer] eut recours à l'Antiquité non seulement pour l'ivresse dionysiaque, mais aussi pour la lucidité apollinienne $»^{23}$.

L'innovation (la provocation) de Nietzsche dans la Naissance de la tragédie consiste à battre en brèche la conception classique de l'Antiquité héritée de Winckelmann pour y voir «l'art dionysien ", bref : «l'orgiasme " ${ }^{24}$. C'est sur cette même reconnaissance que se fonde l'étude warburgiennene de l'œuvre de Dürer, introducteur de la Renaissance dans les pays du Nord. "Jusqu'ici, le classicisme partial et toujours admis de la théorie de la "grandeur tranquille» de l'Antiquité a empêché que l'on se plonge plus sérieusement dans l'examen des documents, et donc que l'on souligne comme il se devait l'enseignement de cette gravure et de ce dessin $»^{25}$. Le dessin de Dürer, La Mort d'Orphée, et la gravure sur cuivre, déjà évoquée, consacrée au même thème (tous deux conservés à la Kunsthalle de Hambourg) nous apprennent que, dès la seconde moitié du XVe siècle, "les artistes italiens ont cherché dans le trésor perdu et retrouvé des formes antiques, autant que la mesure de l'idéal classique, des modèles de mimiques pathétiques fortement accentuées ${ }^{26}$. La thématique de cette gravure mérite d'ailleurs d'être soulignée : Orphée, figure apollinienne, est tué par les ménades de Dionysos qui voulait se venger du culte qu'Orphée rendait à Apollon. Mais lisons plus en détail le commentaire que Warburg lui consacre: "C'est une voix authentiquement antique, familière à la Renaissance, qui retentit ici ; car la Mort d'Orphée n'est pas seulement un motif d'atelier, dont l'intérêt ne serait que formel: c'est aussi une expérience authentique, revécue passionnément et intelligemment, dans l'esprit et selon la lettre de l'Antiquité païenne, et née des sombres mystères de la légende de Dionysos ${ }^{27}$. Le psycho-historien suggère que la Renaissance a compris, oui : revécu l'Antique dans la mesure précisément où elle s'est ouverte à l'ivresse dionysiaque. Lorsque les artistes du Quattrocento imitaient l'Antique, ils n'y voyaient pas un simple répertoire de figures et de gestes où puiser à volonté. Bien plus fondamentalement, ils ré-animaient son 
antagonisme fondamental grâce à «l'aptitude à cet acte esthétique qu'est l'» empathie » en train de devenir une force constituant le style $»^{28}$. C'est à l'occasion notamment des fêtes et des danses, domaine par excellence de Dionysos, que les hommes de la Renaissance revivaient la gestuelle antique et les tensions qui s'y originent. Ces forces ne se limitent ni à l'Antiquité ni à sa Renaissance; tirant les conséquences de l'affirmation de Nietzsche selon laquelle les forces olympiennes et orgiaques sont des "pulsions artistiques de la nature» (N.T., p. 32), c'est-à-dire des constantes ontologiques de l'être, Warburg soutient que leur conflit traverse l'histoire. Lui qui s'est donné pour tâche d'élaborer une "psychologie historique de l'expression humaine $»^{29}$, considère que chez les «clowns » qui surgissent au soir de la cérémonie humiskatcina, on trouve le même dualisme que dans la tragédie grecque. «Pour peu que l'on connaisse un peu la tragédie antique on retrouvera ici la dualité du chœur tragique et du jeu satyrique, «greffés sur une seule branche $»^{30}$.

\section{Polarités}

De ce dualisme de forces psychiques à l'œuvre dans les formules du pathos, j'aimerais encore souligner que, contrairement à ce qu'on pourrait croire, il ne se superpose ni toujours ni exactement avec l'opposition entre Apollon et Dionysos. La note qui suit, rédigée pour la conférence de Kreuzlingen sur le rituel du serpent, montre que les paires conceptuelles warburgiennes ne sont pas strictement équivalentes. «Placé par ma naissance entre l'Orient et l'Occident, poussé par une affinité élective vers l'Italie, qui doit chercher à se construire une nouvelle personnalité autour de la ligne de partage des eaux entre l'Antiquité païenne et la Renaissance chrétienne du XVe siècle, je fus poussé vers l'Amérique, un objet mis au service d'une cause supra-personnelle, pour y connaître la vie dans sa tension entre les deux pôles que sont l'énergie naturelle, instinctive et païenne, et l'intelligence organisée ${ }^{31}$. Ce passage met en exergue une tension schizoïde chez l'individu Aby Warburg, mais aussi dans l'histoire culturelle de l'humanité (l'Italie de la Renaissance, le Nouveau Mexique des Indiens hopis). Les pôles de cette tension sont caractérisés par trois oppositions : entre l'Orient et l'Occident, l'Antiquité païenne et la Renaissance chrétienne, l'énergie naturelle, instinctive et païenne et l'intelligence organisée. Ces trois polarités ne coïncident pas immédiatement avec celle entre Apollon et Dionysos. La première, entre l'Orient et l'Occident, peut tout à fait être référée à celle entre dionysiaque et apollinien. En évoquant «le déchaînement débridé de mouvements expressifs corporels tel qu'il se produisait tout particulièrement en Asie mineure, dans le cortège des dieux de l'ivresse ${ }^{32}$, l'introduction de l'atlas Mnemosyne associe le dionysiaque à l'Orient. Du second conflit, celui entre l'Antiquité païenne et le christianisme, il est plus difficile de considérer qu'il recouvre celui des deux divinités, également païennes. Il est néanmoins possible de les recouper si l'on admet que lorsque Warburg oppose christianisme et Antiquité, il conçoit la Grèce antique comme fondamentalement dionysiaque. Le christianisme peut alors être érigé en antithèse des « commotions orgiaques » (N.T., p. 48) des fidèles de Dionysos. Mais, pour Nietzsche, cette antithèse entre le Christ et Dionysos n'a pas le même sens que celle qui oppose ce dernier au dieu de Delphes, puisque, tandis que celle-ci donne lieu à des réconciliations temporaires mais fertiles, celle-là est antinomique, irréconciliable et absolue ${ }^{33}$. Ecce homo s'achève sur ces mots : "M'a-t-on compris? - Dionysos contre le Crucifié " $^{34}$. La troisième opposition, qui distingue l'» intelligence organisée » de l'» énergie naturelle, instinctive et païenne », 
est plus difficile encore à ramener à celle qui se noue entre les deux dieux. On pourrait caractériser le dionysiaque en termes d'» énergie naturelle » et «instinctive » car, ce qui s'y exprime, c'est « l'abolition du voile de Maya, l'être-un comme génie de l'espèce, ou même comme génie de la nature. C'est l'essence de la nature qui doit ici s'exprimer symboliquement» (N.T., p.35). Quant à la mise en rapport de "l'intelligence organisée " avec Apollon, elle n'est guère évidente, le couple Apollon / Dionysos n'équivalant en aucune façon à la distinction traditionnelle entre raison et passion. Néanmoins, dans la mesure où Apollon est le dieu de la mesure, on pourrait éventuellement l'associer à l'idée d'organisation. Mais cette interprétation peut à juste titre paraitre forcée dans la mesure où le point qui intéresse Warburg dans sa conférence sur les Indiens hopis est l'opposition entre mythologie religieuse et rationalité scientifique, une opposition qui n'intervient pas dans la Naissance de la tragédie. Bien plus, il me semble important de ne pas chercher à superposer à tout prix la série d'opposés warburgiennes au couple nietzschéen d'Apollon et de Dionysos. La tension précédemment évoquée entre la nymphe extatique et le dieu fluvial mélancolique ne s'y superpose pas non plus parfaitement. D'abord, les nymphes peuvent être associées à chacun des deux dieux. On pourrait toutefois essayer de les associer plus étroitement à Dionysos en rappelant l'obsession de Warburg pour la Nymphe que, "remontant jusqu'à son origine païenne, il [...] rapporte aux ménades déchaînées des mystères de l'Antiquité, aux bacchantes porteuses de serpents, ornées de serpents, du dieu sacrifié, Dionysos $»^{35}$. Mais cette association ne peut être tenue absolument: dans les planches 33, 39 et 40 de l'atlas, on voit Apollon et la nymphe Daphné. De l'autre côté, il ne paraît guère pertinent d'identifier le dieu fluvial mélancolique à cet Apollon que Nietzsche qualifie de "brillant» et "solaire». Le propre de ces séries d'oppositions semble donc résider précisément dans le fait qu'elles forment un faisceau dont les extrémités ne se recouvrent pas complètement. Les extrêmes warburgienss se multiplient, oscillent et se déplacent comme - risquons la comparaison - dans un tableau de Balla (Fig.1). 
Fig. 1

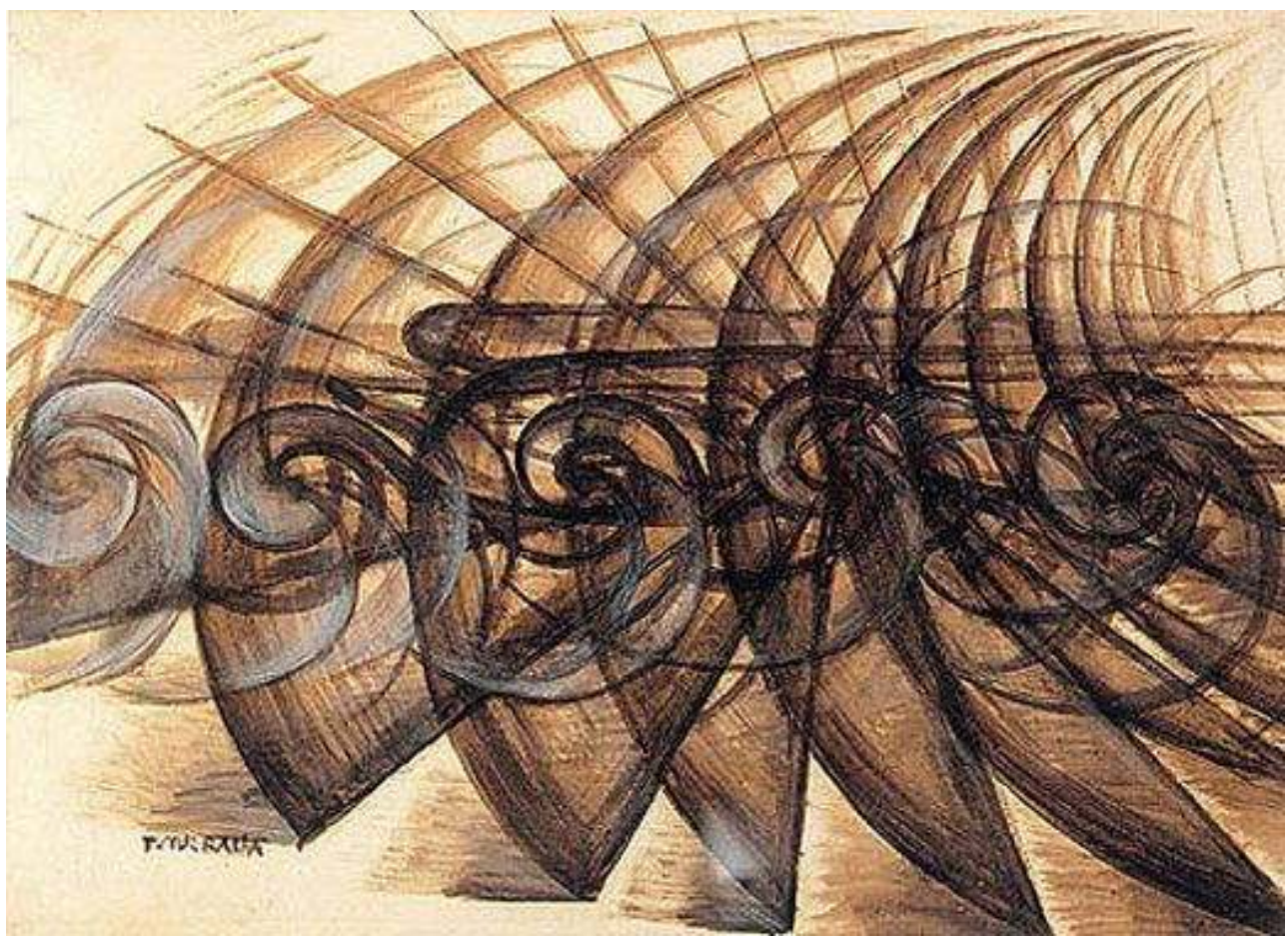

Giacomo Balla, Vélocité d'un motocycle (1913) Oscillations et hybridations, sistole / diastole. Du temps.

12 J'aimerais enfin consacrer quelques remarques à la façon dont Warburg conçoit la relation entre ces tensions opposées. Sa position diffère radicalement de celle de Kant qui pense les rapports entre opposés sur le mode de l'antinomie, de la synthèse ou encore de l'union dans l'horizon d'un idéal régulateur. La solution hégélienne (dialectique) n'est pas pour autant adoptée et c'est une nouvelle fois du côté de Nietzsche qu'il faut se tourner. Celui-ci voit dans les rapports entre Apollon et Dionysos une « mystérieuse union » que l'on peut comprendre par analogie avec la génération.

«L'entier développement de l'art est lié à la dualité de l'apollinien et du dionysiaque comme, analogiquement, la génération - dans ce combat perpétuel où la réconciliation n'intervient jamais que de façon périodique - dépend de la différence des sexes $»^{36}$. La dualité, mieux le duel, entre les deux forces est créateur, puisque c'est à lui qu'on doit «l'entier développement de l'art ». Que la différence soit créatrice peut être compris à partir d'une analogie avec la différence sexuelle qui conditionne toute naissance. Je disais un peu plus haut que l'opposition entre le féminin et le masculin est absente de la paire nietzschéenne formée par Apollon et

Dionysos. On voit ici comment elle pourrait lui être intégrée. Mais revenons à l'analogie entre la génération et la naissance de l'art grec qui donne au titre même de la Naissance de la tragédie une coloration nouvelle. Deux choses encore doivent être notées. La première est que l'union, la réunion est toujours temporaire. Elle relève du miracle. La seconde est que, dans la mesure où le conflit est la condition de possibilité de l'union, il est nécessaire qu'il soit perpétué ${ }^{37}$.

Mais la façon dont Warburg pense les rapports entre Apollon et Dionysos, et plus généralement entre tensions psychiques opposées, est différente. - Quant à l'hypothèse de Nietzsche selon laquelle le conflit psychique repose en dernière instance sur un 
conflit métaphysique, elle n'est pas développée. - C'est en termes d'oscillations que le rapport entre les pôles antagonistes est décrit. Comme chez Hegel, la pensée d'un rapport entre les opposés implique une refonte du concept de temps. Mais en considérant le mouvement de l'esprit et de l'histoire en termes de battement - l'homme de la Renaissance, par exemple, était « conscient d'osciller entre deux pôles, de tendre vers un équilibre nouveau $»^{38}$-, Warburg se distingue radicalement de Hegel tout comme de Cassirer et de Panofsky qui conçoivent le cours du temps de façon téléologique, chronologique et dialectique. Le mouvement oscillatoire de l'esprit et du temps, tel qu'il est compris par Warburg, se donne à voir dans le passage suivant. "J'ai observé chez les Indiens deux processus juxtaposés, qui montrent de façon étonnamment pertinente la polarité de l'être humain luttant contre la nature. D'abord la volonté de maitrise magique de la nature par la métamorphose en animaux, et, en second lieu, la faculté de comprendre la nature, dans une démarche pertinente d'abstraction, de façon cosmique et architectonique comme un tout objectivement cohérent et déterminé tectoniquement $»^{39}$. Ces deux pôles que sont l'énergie naturelle, instinctive et païenne, et l'intelligence organisée sont, en dépit de leur apparente contradiction, « juxtaposés ", posés l'un à côte de l'autre, de sorte que l'homme passe de l'un à l'autre, constamment. À ce modèle de l'oscillation, du battement (cardiaque) « entre systole et diastole $\aleph^{40}$, d'un battement vivant, vital, de dilation puis de contraction, à ce modèle, enfin, du cycle - Warburg note l'existence de « phénomènes de cycles dans l'alternance des formes d'expression artistiques $»^{41}-$ s'ajoute celui de la croissance conjointe des contraires, c'est-à-dire de l'hybride comme croisement de deux individus d'espèces, de races ou de variétés différentes. "L'époque où, comme l'écrit Jean Paul, la logique et la magie "fleurissaient, greffées sur un même tronc", comme le trope et la métaphore, est en fait de tous les temps $»^{42}$. La réunion féconde, la floraison conjointe de la magie et de la logique est évidemment impensable dans une perspective kantienne où la critique a précisément pour fonction de tracer les limites entre ce qui relève de l'entendement ou de la raison et ce qui n'en relève pas. Pour elle, le logique et le magique sont non seulement distincts mais exclusifs l'un de l'autre - seul le mythe religieux peut être récupéré à des fins pratiques. Bien plus, si la magie consiste à croire que la pensée peut agir sur les êtres et que des pratiques empiriques peuvent influer sur le suprasensible, elle constitue l'un des plus graves errements que l'Aufklärung doit dénoncer, l'émancipation de la raison passant par son éradication. Enfin, concernant les schémas temporels impliqués par une mise en rapport des opposés, il convient de remarquer qu'en déclarant que l'esprit oscille du mythique au scientifique et inversement, Warburg ruine la croyance au progrès. Tout à fait étrangère lui est l'idée d'un progrès de la raison pure et la question de savoir si, du point de vue transcendantal, - je cite la Critique de la raison pure - « ce que tant de siècles n'ont pu exécuter ne pourrait pas être accompli avant la fin de celui-ci, c'est-à-dire si l'on ne pourrait pas satisfaire entièrement la raison humaine dans une matière qui a toujours, mais inutilement jusqu'ici, occupé son désir de savoir $»^{43}$. En affirmant l'existence de "l'identité ou plutôt [de] l'indestructibilité de l'homme primitif qui demeure éternellement le même à toutes les époques $»^{44}$, Warburg écarte toute idée de progrès mais aussi toute téléologie de l'esprit, de l'histoire ou de la culture. Le mythe n'est ni l'enfance de l'individu ni l'état primitif de l'humanité, il est un pôle permanent de l'esprit et de la culture. L'époque où la logique et la magie "fleurissaient, greffées sur un même tronc " n'est d'aucun temps. Ni d'aucun lieu, puisqu'on retrouve cette structure schizophrène dans la Grèce antique, dans la Florence de la Renaissance ou encore, à la fin du XIXe 
siècle, au Nouveau-Mexique, chez les Indiens pueblos. « C'est un vieux livre à feuilleter - Athènes and the Indians - rien que des cousins $»^{45}$. Il faudrait, pour être complet, ajouter que, du point de vue de la liberté, Warburg ne met pas sur le même plan la raison et la magie ou, plus précisément, l'astrologie. Il dénonce «la superstition astrologique ", " cette redoutable ennemie de la création artistique libre ", cette «idolâtrie du destin [...] barbare », et salue en Luther et en Dürer les artisans de « la lutte pour la libération intérieure, intellectuelle et religieuse, de l'homme moderne $»^{46}$. La conquête de la liberté de pensée semble bien être une valeur qui échappe à la relativité des oscillations et à la contingence de la réussite d'une greffe. Lisons une dernière citation : "Nous sommes au siècle de Faust, où le savant moderne - à mi-chemin entre la pratique magique et la cosmologie mathématique - cherchait à conquérir l'espace de la réflexion intellectuelle entre l'objet et lui. Car il faut sans cesse reconquérir Athènes depuis Alexandrie [Athen will eben immer wieder neu aus Alexandrien zurückerobert sein]. De ce point de vue, les images et les paroles dont il a été question ici doivent être considérées un peu comme des documents d'archives encore inexplorés qui témoignent de l'histoire tragique de la liberté de pensée de l'Européen moderne $\aleph^{47}$. Considérer le savant moderne comme un Faust, la science contemporaine (nous sommes en 1919) comme un mélange de pratiques magiques et de cosmologie mathématique est pour le moins surprenant et indique la défiance de Warburg envers le positivisme ainsi que son scepticisme à l'égard d'une société matérialiste. "La civilisation de l'âge mécanique, écrit-il ailleurs, détruit [...] ce que la connaissance de la nature, née du mythe, avait péniblement construit, l'espace de contemplation qui est devenu l'espace de pensée $»^{48}$. Mais la conquête d'un espace intellectuel contre la superstition n'en est pas moins saluée et souhaitée. Que son avènement désiré ne soit pas intégré à un mouvement téléologique ou dialectique montre une nouvelle fois la position inédite de Warburg dans un horizon néokantien dont on peut pourtant considérer qu'il partage certaines des préoccupations : penser, dans le temps, les pôles séparés par l'entendement. L'idée d'un va-et-vient entre eux semble néanmoins entrer en tension avec l'exigence de liberté qui aimerait voir battre leur mouvement dans une certaine direction. Si cette exigence n'empêche pas de considérer les opposés et la temporalité en termes de battement ou de greffe, elle fait néanmoins de l'oscillation et de la croissance simultanée des mouvements tragiques.

\section{NOTES}

1. A. Warburg, "Albert Dürer et l'Antiquité italienne ", in A. Warburg, Essais florentins et autres textes, trad. S. Muller, Paris, Klincksieck, 1990, p. 161.

2. A. Warburg, «L'art du portrait et la bourgeoisie florentine », in Essais florentins, op. cit., p. 110. Ce refus de l'alternative caractérise l'homme de la Florence des Médicis, à la recherche d'un compromis artistique entre l'Église et le monde, le passé antique et le présent chrétien.

3. Voir G. W. F. Hegel, Encyclopédie des sciences philosophiques, vol. I. "Science de la logique », éd. et trad. B. Bourgeois, Paris, Vrin, 1970, § 55, p. 317. Hegel vise essentiellement Kant. 
4. B. Bourgeois, "Présentation de la Science de la Logique ", in G. W. F Hegel, Encyclopédie des Sciences Philosophiques, vol. 1, op. cit., p. 14.

5. E. Cassirer, « Le concept de forme symbolique dans l'édification des sciences de l'esprit », in $\mathrm{E}$. Cassirer, Trois essais sur le symbolique, trad. J. Carro et J. Gaubert, Paris, Éditions du Cerf, 1997, p. 13.

6. A. Warburg, « Art italien et astrologie internationale au palais Schifanoia de Ferrare ", in Essais florentins, op. cit., p. 199.

7. A. Warburg, « Albert Dürer et l'Antiquité italienne », p. 161.

8. G. W. F. Hegel, Phénoménologie de l'esprit, trad. B. Bourgeois, Paris, Vrin, 1997, préface, § 56, p. 139.

9. Ibid., § 20, p. 69 et 71.

10. E. Cassirer, "Éloge funèbre du professeur Aby M. Warburg ", in E. Cassirer, Écrits sur l'art, trad. C. Berner, F. Capeillères, J. Carro et J. Gaubert, Paris, Éditions du Cerf, 1995, p. 55.

11. Pour l'expression d' " exagération gestuelle », voir " Albert Dürer et l'Antiquité italienne », p. 165.

12. A. Warburg, «L'entrée du style idéal antiquisant dans la peinture du début de la Renaissance ", in Essais florentins, p. 224.

13. A. Warburg, notes du 3 avril 1929, Tagebuch der Kulturwissenschaftlichen Bibliothek Warburg, 7, 249. Cité par Philippe-Alain Michaud, Aby Warburg et l'image en mouvement, Paris, Macula, 1998, p. 234.

14. F. Nietzsche, La Naissance de la tragédie ou Hellénité et pessimisme, trad. M. Haar, Ph. LacoueLabarthe et J.-L. Nancy, Paris, Gallimard, 1977, « Essai d'autocritique », p. 14-15.

15. A. Warburg, « Albert Dürer et l'Antiquité italienne », p. 164.

16. A. Warburg, «Souvenirs d'un voyage en pays pueblo. Notes inédites pour la conférence de Kreuzlingen sur « Le rituel du serpent » (1923) », citées par Ph.-A. Michaud, Aby Warburg et l'image en mouvement, op. cit., Annexe I, p. 266.

17. Ibid., Annexe II, p. 282.

18. A. Warburg, « Albert Dürer et l'Antiquité italienne », p. 162.

19. Les expressions et citations de Nietzsche sont, sauf mention contraire, tirées du début de $L a$ Naissance de la Tragédie (notée N.T.), des pages 28 à 42.

20. F. Nietzsche, La Naissance de la tragédie, p.41-42; A. Warburg, «L'entrée du style idéal antiquisant dans la peinture du début de la Renaissance ", p. 241-242.

21. F. Nietzsche, La Généalogie de la morale, trad. É. Blondel, O. Hansen-Løve, T. Leydenbach, P. Pénisson, Paris, Flammarion, 1996, p. 64. Mais la Renaissance fait l'objet d'évaluations diverses, seul étant unique l'angle d'attaque axiologique de Nietzsche. Voir sur ce point T. Gontier, "Nietzsche, Burckhardt et la « question » de la Renaissance », Noesis [En ligne], No 10 | 2006, mis en ligne le 02 juillet 2008. URL: http://noesis.revues.org/index422.html qui décline les différentes alliances et oppositions dans lesquelles la Renaissance est prise (la Wiedergeburt wagnérienne, le socratisme, la Réforme, les Lumières, la modernité). «Les camps changent sans remettre en cause, encore une fois, la constance du projet » (§ 14).

22. A. Warburg, «La divination antique et païenne dans les écrits et les images à l'époque de Luther ", in Essais florentins, p. 268.

23. A. Warburg, « Albert Dürer et l'Antiquité italienne », p. 164

24. Voir F. Nietzsche, Le crépuscule des idoles, in Euvres philosophiques complètes, VIII-1, éd. G. Colli et M. Montinari, Paris, Gallimard, 1977, p. 149-150. «C'est moi qui, le premier, pour mieux comprendre l'instinct hellénique archaïque, encore riche, et même débordant, ai pris au sérieux cet extraordinaire phénomène qui porte le nom de Dionysos : il ne s'explique que par un tropplein de forces » (ibid., p. 148-149).

25. A. Warburg, « Albert Dürer et l'Antiquité italienne », p. 161.

26. Ibid. 
27. Ibid., p. 162

28. A. Warburg, Avant-propos à «La Naissance de Vénus et Le Printemps de Sandro Botticelli. Étude sur les représentations de l'Antiquité au début de la Renaissance italienne ", in Essais florentins, p. 49.

29. A. Warburg, « Art italien et astrologie internationale au Palazzo Schifanoia à Ferrare », p. 215. 30. A. Warburg, Le rituel du serpent. Récit d'un voyage en pays pueblo, trad. D. Bodart, P. Guiton et S. Muller, Paris, Macula, 2003, p. 101.

31. A. Warburg, "Souvenirs d'un voyage en pays pueblo. Notes inédites pour la conférence de Kreuzlingen sur «Le rituel du serpent » (1923)», in Ph.-A. Michaud, Aby Warburg et l'image en mouvement, Annexe II, p. 282.

32. A. Warburg, Der Bilderatlas Mnemosyne [1929], in Gesammelte Schriften, II-1, éd. M. Warnke et C. Brink, Berlin, Akademie Verlag, 2000, Introduction, p. 4. C'est moi qui traduit.

33. Voir l'Introduction d'E. Blondel à F. Nietzsche, L'Antéchrist, Paris, Flammarion, 1994, p. 34 où il remarque que, dans L'Antéchrist, Nietzsche évoque "une fêlure, mais gigantesque, entre le Crucifié et Dionysos, entre le Non et le Oui, entre la décadence, le ressentiment et la belle humeur, entre Luther (ou Paul) et Nietzsche ».

34. F. Nietzsche, Ecce homo, "Warum ich ein Schicksal bin ». La section (et le livre) s'achèvent par ces mots : «Hat man mich verstanden? - Dionysos gegen den Gekreuzigten... ». Voir aussi La Naissance de la tragédie, "Essai d'autocritique», p. 18 qui voit dans cet essai de jeunesse " une contredoctrine et une contre-évaluation de la vie, purement artistique, anti-chrétienne. Mais comment la nommer ? En philologue, en homme du langage, je la baptisai non sans quelque liberté - mais qui saurait au juste le nom de l'antéchrist? - du nom d'un dieu grec ? je l'appelai dionysiaque ».

35. J. L. Koerner, Introduction à A. Warburg, Le rituel du serpent, p. 34.

36. F. Nietzsche, La Naissance de la tragédie, p. 27. Il est question de cette "mystérieuse union » p. 42.

37. Sur ces conflits et enfantements réciproques miraculeux, voir aussi La Naissance de la tragédie, p. 27-28. «Ces deux impulsions si différentes marchent de front, mais la plupart du temps en conflit ouvert, s'excitant mutuellement à des productions toujours nouvelles et de plus en plus vigoureuses afin de perpétuer en elles ce combat de contraires (entre lesquels le mot "art » qu'on leur attribue en commun ne fait qu'apparemment jeter un pont), jusqu'à ce qu'enfin, par un geste métaphysique miraculeux de la "volonté » hellénique, elles apparaissent accouplées l'une à l'autre et, dans cet accouplement, en viennent à engendrer l'œuvre d'art à la fois dionysiaque et apollinienne, la tragédie attique ».

38. A. Warburg, «Les dernières volontés de Francesco Sassetti », in Essais florentins, p. 185. Il s'agit pour Francesco Sassetti de trouver un compromis entre la confiance médiévale en Dieu et la confiance en soi de l'homme de la Renaissance.

39. A. Warburg, Notes inédites pour la conférence de Kreuzlingen sur «Le rituel du serpent ", citées par Ph.-A. Michaud, Aby Warburg et l'image en mouvement, Annexe I, p. 270.

40. Ibid., Annexe I, p. 273.

41. A. Warburg, « Albert Dürer et l'Antiquité italienne », p. 165.

42. A. Warburg, «La divination antique et païenne dans les écrits et les images à l'époque de Luther », p. 251.

43. E. Kant, Critique de la raison pure, édition F. Alquié, trad. A. J.-L. Delamarre et F. Marty à partir de la traduction de J. Barni, Paris, Gallimard, 1980, IIe partie: «Théorie transcendantale de la méthode », chapitre $4:$ « Histoire de la raison pure », p. 708.

44. A. Warburg, Notes inédites pour la conférence de Kreuzlingen sur "Le rituel du serpent », citées par Ph.-A. Michaud, op. cit., Annexe I, p. 255.

45. A. Warburg, On planned American visit (1927), cité par Ph.-A. Michaud, op. cit., Annexe II, p. 281.

46. A. Warburg, «La divination antique et païenne dans les écrits et les images à l'époque de Luther ", p. 199, p. 211 et p. 282 pour cette qualification de la superstition. 
47. Ibid., p. 285.

48. A. Warburg, Le rituel du serpent, p. 132-133.

INDEX

Mots-clés : Sujet, objet, conciliation des opposés, formules du pathos, formes symboliques

\section{AUTEUR}

\section{AUDREY RIEBER}

Agrégée et docteur en philosophie, elle effectue depuis 2009 un postdoctorat au sein du pôle de recherche eikones NFS Bildkritik (Bâle, Suisse). Elle est également professeur invitée du Master " Aisthesis " (KU Eichstâtt, Allemagne). Elle a notamment publié Art, histoire et signification. Un essai d'épistémologie d'histoire de l'art autour de l'iconologie d'Erwin Panofsky (L'Harmattan, 2012), cotraduit l'ouvrage de Hans Belting, Florence et Bagdad. Une histoire du regard entre Orient et Occident (Gallimard, 2012), et dirigé le dernier numéro de la revue Appareil (« Penser l’art, penser l'histoire ", n 9, juillet 2012). 